\title{
On TP-AGB stars and the mass of galaxies
}

\author{
A. Gustavo Bruzual ${ }^{1}$ \\ ${ }^{1}$ CIDA, AP 264, Mérida, Venezuela \\ email: bruzual@cida.ve
}

\begin{abstract}
Recent calculations of evolutionary tracks of TP-AGB stars of different mass and metallicity by Marigo et al. (2007) have been incorporated in the Bruzual \& Charlot evolutionary population synthesis models. The mass of the stellar population in HUDF galaxies at $z$ from 1 to 3 determined from fits to the spectro-photometric data of these galaxies is 50 to $80 \%$ lower than the mass determined from the $\mathrm{BC} 03$ models. The ages inferred for these populations are, with exceptions, 40 to $60 \%$ of the BC03 estimates.
\end{abstract}

\section{Introduction}

In the oral presentation of this paper I gave a summary of the current status of various high resolution stellar spectral libraries and their use in population synthesis models. A written version of this summary can be found in a series of conference papers appeared in recent years, (Bruzual 2004, 2005, 2006) and will not be repeated here for reasons of space. In this paper I will concentrate in the second part of my talk which versed on the rôle of the thermally pulsing asymptotic giant branch (TP-AGB) phase of stellar evolution on the integrated properties of stellar populations.

It has been pointed out by several authors, e.g. Maraston et al. (2006), Kannappan \& Gawiser (2007), that the estimates of the age and mass of the stellar population present in a galaxy depend critically on the ingredients of the stellar population model used to fit the galaxy spectrum. Maraston et al. (2006) have shown that the treatment of the TP-AGB phase of stellar evolution is a source of major discrepancy in the determination of the spectroscopic age and mass of high-z $(1.4<z<2.7)$ galaxies. The mid-UV spectra of these galaxies indicate ages in the range from $0.2-2 \mathrm{Gyr}$, at which the contribution of TP-AGB stars in the rest-frame near-IR sampled by Spitzer is expected to be at maximum. Maraston et al. (2006) find that in general the Maraston (2005) models (M05 hereafter) provide better fits to the observations than the Bruzual \& Charlot (2003) models, hereafter BC03, and other models available in the literature, and indicate systematically lower ages and, on average, $60 \%$ lower masses for the stellar populations sampled in these galaxies. According to Maraston et al. (2006) the source of this discrepancy is primarily a consequence of the different treatment of the TP-AGB phase in the evolutionary models.

Marigo et al. (2007) have recently concluded new calculations of the TP-AGB evolutionary phase for stars of different mass and metallicity. The evolution of the stars is now computed accounting for the changes in the chemical composition of the envelopes. As a consequence of this prescription, the signature of TP-AGB stars around 1 Gyr, i.e the red color of the integrated stellar population, becomes more relevant than in previous computations. The full implementation of the new stellar libraries and the Marigo et al. (2007) TP-AGB evolutionary tracks in population synthesis models is in preparation by Charlot \& Bruzual (2007, CB07 hereafter). All the BC03 and CB07 SSP models shown in this paper have been computed for the Chabrier (2003) IMF and the Westera et al. (2002) stellar library. Fig. 1 shows the time dependence of the fraction of the galaxy 

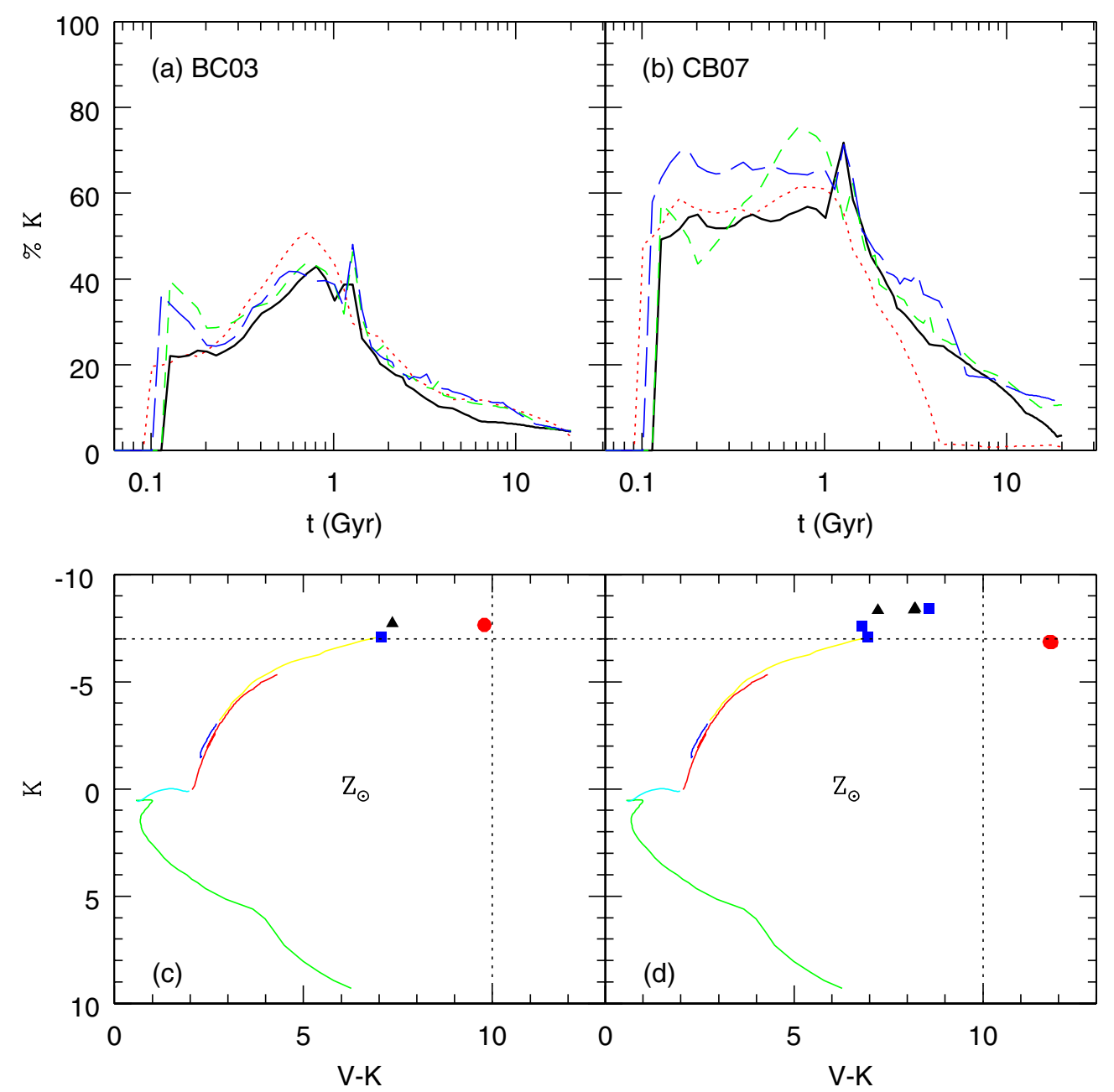

Figure 1. Top frames: time dependence of the fraction of the galaxy luminosity emitted in the K-band according to $(a)$ the BC03 and (b) the CB07 SSP models. Different lines correspond to different metallicities of the stellar population, as follows: $Z_{\odot}$ (black solid line), $0.4 \times Z_{\odot}$ (green long-dashed line), $0.2 \times Z_{\odot}$ (blue short-dashed line), $2.5 \times Z_{\odot}$ (red dotted line). All models shown in this figure were computed for the Padova 1994 tracks assuming the Chabrier (2003) IMF. Figures drawn in color are available in the electronic version of this paper. Bottom frames: 1.3 Gyr $Z_{\odot}$ isochrone computed using $(c)$ the BC03 and $(d)$ the $\mathrm{CB} 07$ models. The lines representing the MS, SGB, RGB, HB, and AGB are identical in both cases, and correspond to the Padova 1994 tracks. The symbols represent the O-rich (blue squares), the C-rich (black triangles), and the superwind (red circles) phases of the TP-AGB. The dotted lines are drawn to guide the eye.

luminosity emitted in the K-band according to the BC03 and CB07 models. From the top frames of Fig. 1 it is apparent that the TP-AGB stars in the CB07 models contribute at least a factor of two more light in the $K$-band than in the BC03 models, which use a different prescription for the TP-AGB evolution (see BC03 for details). At maximum, the TP-AGB contributes close to $70 \%$ of the K-light in the CB07 model but only $40 \%$ in the BC03 model. The peak emission in the BC03 model occurs at around 1 Gyr whereas 


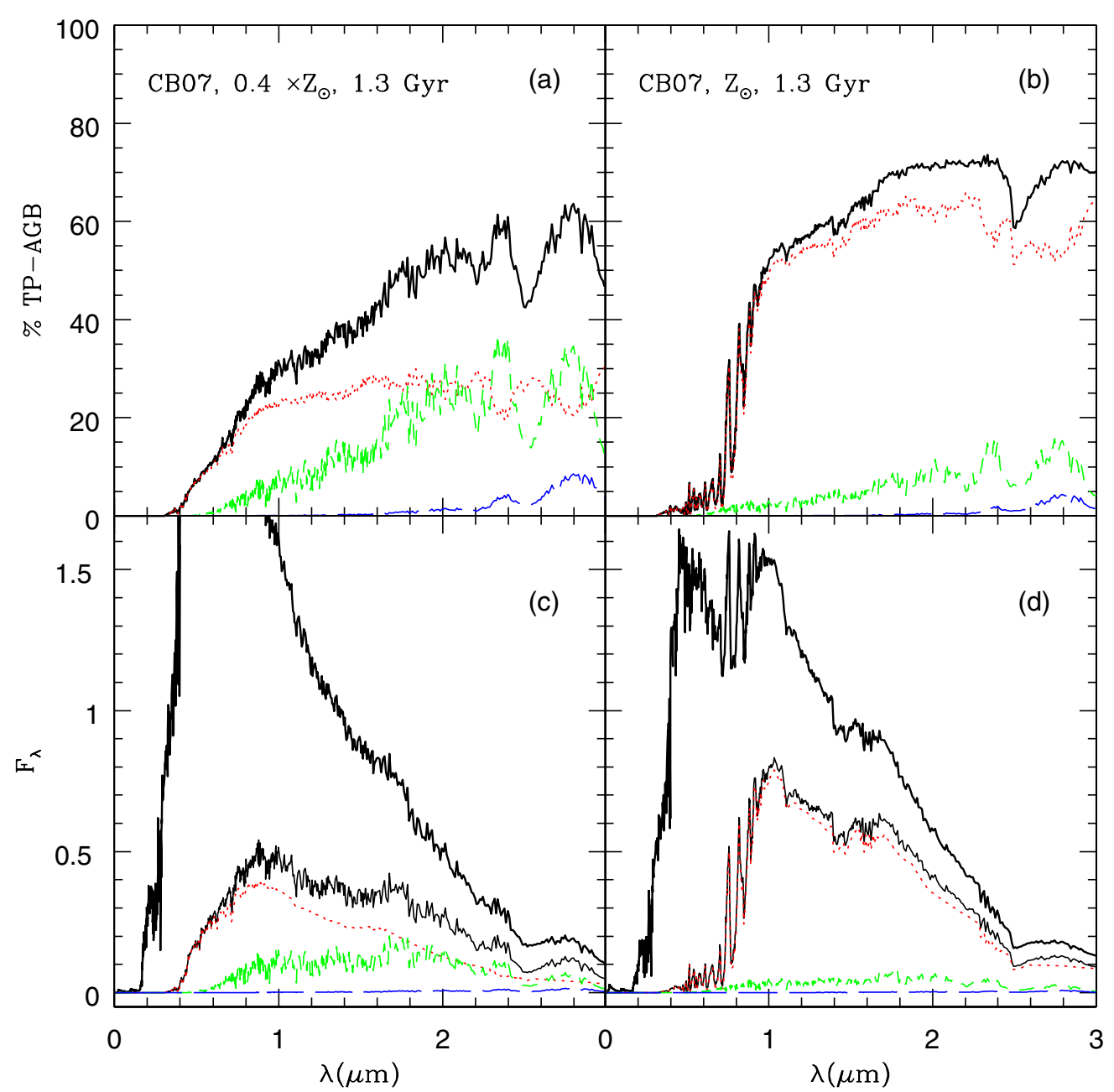

Figure 2. Top frames: Percentage of the luminosity of the 1.3 Gyr SSP emitted by TP-AGB stars as a function of wavelength (heavy solid-line) according to the CB07 models for (a) $Z=0.4 \times Z_{\odot}$, and (b) $Z=Z_{\odot}$. The contribution of the O-rich TP-AGB stars (red dotted-line), the C-rich TP-AGB stars (green short-dashed line), and the TP-AGB stars in the superwind phase (blue long-dashed line) is also indicated. Figures drawn in color are available in the electronic version of this paper. Bottom frames: spectral energy distribution of the 1.3 Gyr SSP as a function of wavelength (heavy solid-line) according to the CB07 models for (c) $Z=0.4 \times Z \odot$, and (d) $Z=Z_{\odot}$. The contribution of all the TP-AGB stars (thin solid-line), the O-rich TP-AGB stars (red dotted-line), the C-rich TP-AGB stars (green short-dashed line), and the TP-AGB stars in the superwind phase (blue long-dashed line) is also indicated.

in the CB07 model it stays high and close to constant from 0.1 to 1 Gyr. To explore the cause of this difference, I plot in the bottom frames of Fig. 1 the 1.3 Gyr $Z=Z_{\odot}$ isochrone for both sets of models. This age corresponds to the spike seen in the $Z=Z_{\odot}$ line in the top frames of this figure. Whereas the Marigo et al. (2007) tracks used in CB07 account for 9 evolutionary stages in the TP-AGB (three in the O-rich phase, three in the C-rich phase, and three in the superwind phase), the BC03 models include only 1 
evolutionary stage on each of these phases. The TP-AGB stars in the CB07 isochrone are close to 1 magnitude brighter in $K$ and reach values of $V-K$ several magnitudes redder than their counterparts in $\mathrm{BC} 03$. The evolutionary rate is such that the total number of TP-AGB stars present in the CB07 1.3 Gyr isochrone is 4 times larger than the number of these stars present in the BC03 models. The TP-AGB stars represent $0.016 \%$ of the total number of stars present in this population at this age in the CB07 model, but only $0.004 \%$ in the BC03 model. The net effect of all these factors is the increased contribution shown in Fig. 1.
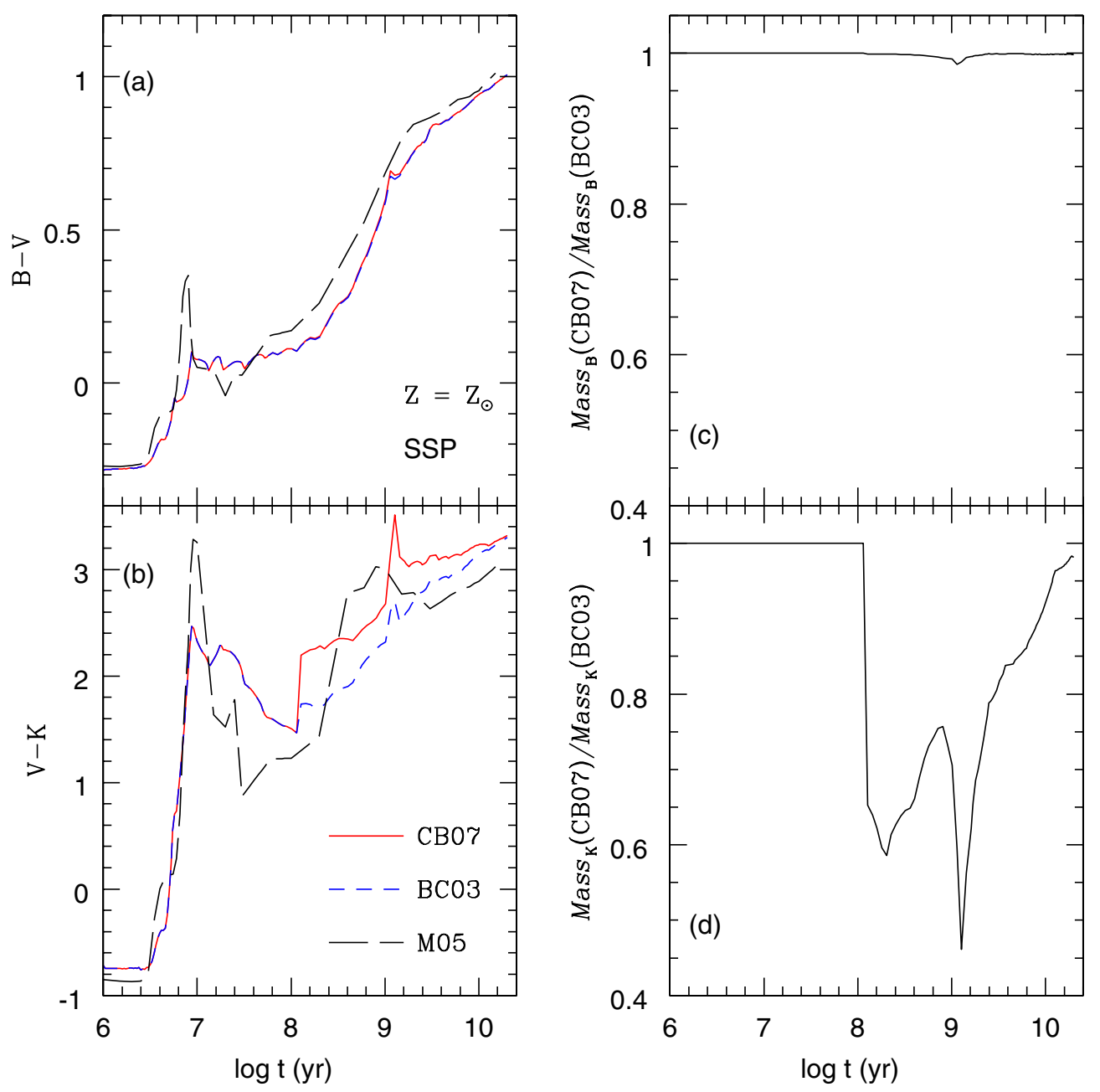

Figure 3. Comparison of (a) the B-V and (b) V-K color evolution of the CB07 models (red solid line) with the BC03 (blue short-dashed line) and the M05 (black long-dashed line) models. Figures drawn in color are available in the electronic version of this paper. (c) and $(d)$ : ratio of the stellar mass determined from the CB07 and the BC03 models for a given $B$ and $K$-band galaxy luminosity, respectively. All models shown in this figure correspond to a $Z=Z_{\odot}$ SSP computed for the Chabrier (2003) IMF. 


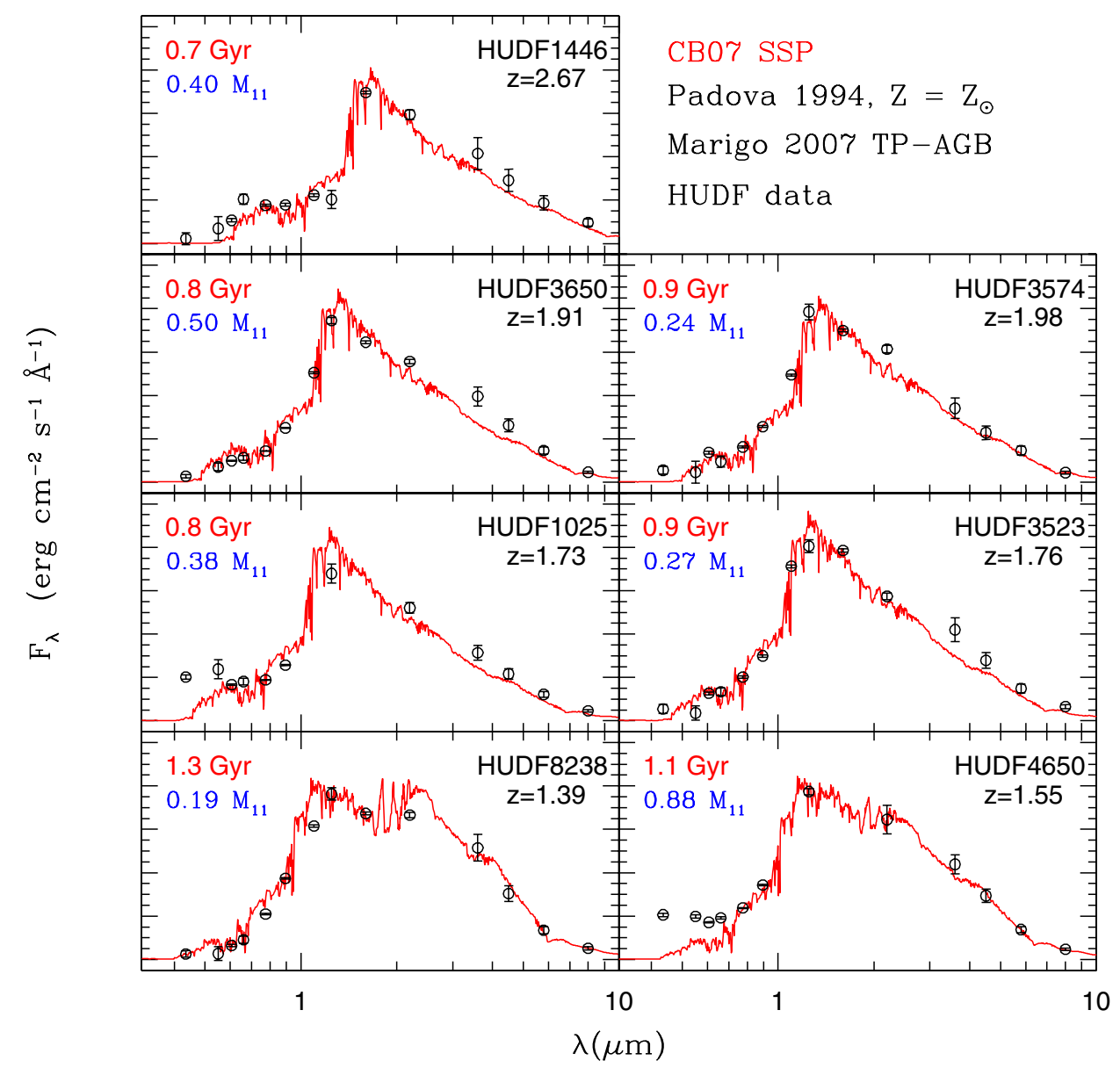

Figure 4. Observed spectral energy distribution (symbols with error bars) of 7 HUDF galaxies taken from Table 1 of Maraston et al. (2006) shown together with the best fitting CB07 $Z=Z_{\odot}$, Chabrier IMF SSP model (red solid-line). The galaxy HUDF identification number, redshift, age, and mass (in units of $10^{11} \mathrm{M}_{\odot}$ ) are given inside each frame and listed in Table 1. For clearness, after determining the galaxy mass the spectra were forced to share the same vertical scale. Figures drawn in color are available in the electronic version of this paper.

Fig. 2 shows the contribution of TP-AGB stars to the spectral energy distribution of the 1.3 Gyr SSP as a function of wavelength according to the CB07 models for $Z=0.4 \times Z_{\odot}$, and $Z=Z_{\odot}$. The relative contribution of the O-rich vs. the C-rich TP-AGB stars depends critically on the assumed stellar metallicity. Fig. 3 compares the $B-V$ and $V-K$ color evolution of the CB07, BC03 and M05 models. In $B-V$ the CB07 and BC03 models are identical at all ages. At early and late ages both sets of models have the same $V-K$ color, but at intermediate ages the CB07 models are considerably redder than the BC03 models. At late ages, the BC03 and CB07 models match very well the observations of nearby early-type galaxies, whereas the M05 models are too blue. The stellar mass determined from the $K$-band luminosity using the CB07 model can be up to $50 \%$ lower than the mass determined from the BC03 model at the same age. The BC03 and CB07 models will predict the same galaxy mass if the $B$-band luminosity is used. 
Table 1. Spectroscopic age and mass ${ }^{3}$ of HUDF galaxies

\begin{tabular}{lccccccc}
\hline HUDF & $z$ & $\begin{array}{c}\mathrm{CB07}^{1} \\
\mathrm{t}(\mathrm{Gyr})\end{array}$ & $\begin{array}{c}\mathrm{CB07}^{1} \\
\mathrm{M}_{11}\end{array}$ & $\begin{array}{c}\mathrm{BC03}^{2} \\
\mathrm{t}(\mathrm{Gyr})\end{array}$ & $\begin{array}{c}\mathrm{BC03}^{2} \\
\mathrm{M}_{11}\end{array}$ & $\begin{array}{c}\mathrm{M} 6^{2} \\
\mathrm{t}(\mathrm{Gyr})\end{array}$ & $\begin{array}{c}\mathrm{M}^{2} \\
\mathrm{M}_{11}\end{array}$ \\
\hline 1446 & 2.67 & 0.72 & 0.40 & 2.30 & 0.80 & 0.70 & 0.40 \\
3650 & 1.91 & 0.81 & 0.50 & 2.30 & 1.60 & 0.50 & 0.65 \\
1025 & 1.73 & 0.81 & 0.38 & 1.70 & 1.25 & 1.70 & 1.00 \\
8238 & 1.39 & 1.28 & 0.19 & 2.30 & 1.13 & 1.00 & 0.46 \\
3574 & 1.98 & 0.90 & 0.24 & 2.60 & 0.53 & 2.30 & 0.56 \\
3523 & 1.76 & 0.90 & 0.27 & 2.30 & 1.00 & 1.40 & 0.60 \\
4650 & 1.55 & 1.10 & 0.88 & 1.70 & 3.50 & 1.70 & 2.20 \\
\hline
\end{tabular}

${ }^{1}$ Determined from fitting a $Z=Z_{\odot}$, Chabrier IMF SSP to the magnitudes in Table 1 of Maraston et al. (2006).

${ }^{2}$ Values read from Fig. 4 of Maraston et al. (2006) assuming $E(B-V)=0$.

${ }^{3}$ The galaxy mass $\mathrm{M}_{11}$ is given in units of $10^{11} \mathrm{M}_{\odot}$.

Fig. 4 compares the observed spectral energy distribution of 7 HUDF galaxies with the best fitting CB07 $Z=Z_{\odot}$, Chabrier IMF SSP model. The fits look quite reasonable and can be improved assuming different star formation histories (e.g. HUDF1025 and HUDF4650) or dust content. The galaxy HUDF identification number, redshift, and derived age and mass (in units of $10^{11} \mathrm{M}_{\odot}$ ) are given inside each frame and listed in Table 1. As expected from the previous discussion, the mass and age of the stellar population determined from the level of the IR flux observed in these galaxies are considerably lower than the values determined by Maraston et al. (2006) from the BC03 (and M05) models. The masses derived from the CB07 models are 50 to $80 \%$ lower than the BC03 masses. The ages derived from the CB07 models are, with exceptions, 40 to $60 \%$ of those derived from BC03.

\section{Acknowledgements}

I thank Paola Marigo and Leo Girardi for providing their calculations of the TP-AGB evolutionary phase ahead of publication, and Stéphane Charlot for allowing me to show results of a joint paper in preparation.

\section{References}

Bruzual, A., G. 2004, in Proceedings of the IAU Symposium No. 222 "The Interplay Among Black Holes, Stars, and ISM in Galactic Nuclei", eds. T. Storchi-Bergmann, L.C. Ho, and H.R. Schmitt, Cambridge: Cambridge University Press, 121, astro-ph/0701922

Bruzual, A., G. 2005, in Proceedings of the Meeting "Resolved Stellar Populations", eds. D. Valls-Gabaud and M. Chávez, ASP Conf. Ser. (in press) astro-ph/0701907

Bruzual, A., G. 2006, in Proceedings of the Meeting "From Stars to Galaxies: Building the Pieces to Build Up the Universe", eds. A. Vallenari, R. Tantalo, L. Portinari, and A. Moretti, ASP Conf. Ser. (in press) astro-ph/0702091

Bruzual, A., G. \& Charlot, S. 2003, MNRAS, 344, 1000

Chabrier, G. 2003, PASP, 115, 763

Charlot, S. \& Bruzual A., G. 2007, MNRAS, in preparation

Kannappan, S.J., \& Gawiser, E. 2007 ApJ Letters, in press, astro-ph/0701749

Le Borgne, J.-F., Bruzual A., G., Pelló, R., Lançon, A., Rocca-Volmerange, B., Sanahuja, B., Schaerer, D., Soubiran, C., \& Vílchez-Gómez, R. 2003, A\&A, 402, 433

Maraston, C. 2005 MNRAS, 362, 799

Maraston, C., Daddi, E., Renzini, A., Cimatti, A., Dickinson, M., Papovich, C., Pasquali, A., \& Pirzkal, N. 2006, ApJ, 652, 85; (Erratum: 2007, ApJ, 656, 1241) 
Marigo et al. 2007, in preparation

Westera, P., Lejeune, T., Buser, R., Cuisinier, F., \& Bruzual A., G. 2002, A\&A, 381, 524

\section{Discussion}

Kroupa: How could your results on the galaxies (their ages and masses) change if the IGIMF is steeper than the standard IMF?.

BRUZUAL: If the number of intermediate mass stars is reduced as a consequence of having a different IMF, we expect a lower contribution of TP-AGB stars to the galaxy luminosity. I would guess that the estimates of the galaxy mass will be somewhat higher than for the standard IMF. The age estimates could also show a trend towards larger values. The opposite should occur for an increased number of intermediate mass stars in the IMF. This is something that can be easily tested with the population synthesis models.

CERviño: When you use the TP-AGB tracks you compute a mean value, but there will be some scatter (TP-AGB stars show variations of a few magnitudes). Have you estimated how large this scatter is? How do you manage to integrate isochrones when they are a "band" and not a single well defined path? How does the TP-AGB affect the surface brightness fluctuations?

BRuzuAL: The evolutionary tracks for TP-AGB stars computed by Marigo et al. (2007) provide well defined values of $\left(a g e, \log T_{e f f}, M_{B O L}\right)$ at all points along the tracks. We use their results as published. Presumably the aspects you mention have already been taken into consideration by the authors of this paper in their careful comparison with observations of real TP-AGB stars. Our isochrones are thus a narrow line and not a "band". At the moment I am working with Rosa González-López-Lira in a paper where we examine the influence of the new TP-AGB evolutionary tracks on the surface brightness fluctuations.

MARAston: This is not true. There is no scatter.

Bruzual: Good to hear that.

MARAston: What differences do you find between the IUE and the HNGSL spectral libraries?

BRUZUAL: The signal-to-noise-ratio is considerably higher in the HNGSL data than in the IUE spectra. As a consequence, the spectral features, absorption lines and discontinuities are much sharper and better defined. No doubt, the HNGSL is more appropriate than the IUE data to study line strength indices in the UV

Gustafsson: You mentioned that your high-resolution indices are quite sensitive to the degree of degrading due to the stellar velocity dispersion. I suppose that in a real galaxy different types of stars may have different velocity dispersion. Is this a worry?

Bruzual: This is certainly a worry when modeling indices like $I_{200}$ and $I_{275}$ defined by Vazdekis \& Arimoto (1999, ApJ, 525, 144), which are very sensitive to the stellar velocity dispersion. Models shown in Bruzual (2006) assume that all the stars share the same value of the velocity dispersion whereas in reality one should consider the dynamics of different stellar groups and use the corresponding $\sigma$ for each kind of stars. 
HAN: In your second conclusion, you said that there is room for improvement in UV. We know that UV in old stellar populations is from hot subdwarfs (EHB). We have a binary model for the formation of hot subdwarfs and the model is well established for galactic hot subdwarfs. We applied the model to evolutionary population synthesis study and proposed a binary model for the UV-upturn of elliptical galaxies (Poster V.10)

BRUZUAL: This is certainly an interesting approach. Binary star evolution is missing from most, if not all, stellar population models. The source of the UV in old populations is not well established yet. Besides EHB stars in some galaxies there is residual star formation. In my talk I referred to the improvement that is needed in the UV spectral libraries, but for sure we also need better evolutionary schemes.

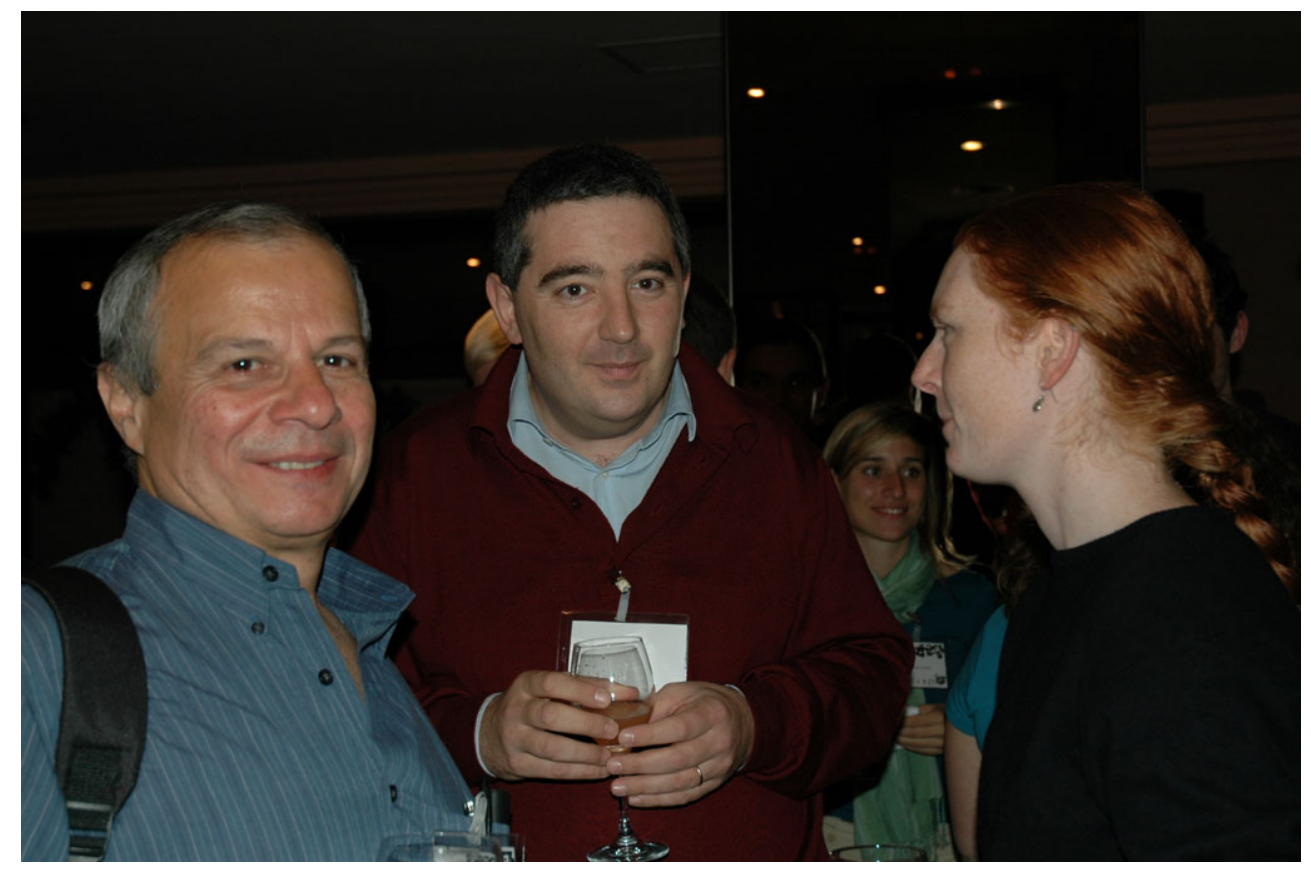

The speaker (left), Stéphane Charlot and Vivienne Wild. 